\title{
Short course hypofractionated whole breast irradiation after conservative surgery: a single institution phase II study
}

\author{
Paola Pinnarò ', Carolina Giordano ${ }^{1}$, Alessia Farneti ${ }^{1}$, Adriana Faiella', Giuseppe laccarino², Valeria Landoni², \\ Diana Giannarelli ${ }^{3}$, Patrizia Vici ${ }^{4}$, Lidia Strigari ${ }^{2}$ and Giuseppe Sanguineti ${ }^{{ }^{*}}$
}

\begin{abstract}
Background: To assess the oncologic outcomes of hypofractionated whole breast irradiation (Hypo-WBI).

Methods: Eligible patients had undergone breast conservative surgery for early breast cancer (pTis-2) and none/limited nodal involvement. Hypo-WBI consisted of 34 Gy in 10 daily fractions over 2 weeks to the whole breast three-dimensional conformal radiotherapy (3DCRT), followed by a single fraction of $8 \mathrm{~Gy}$ to the tumor bed after 1 week (electrons). Primary endpoint is freedom from ipsilateral breast tumor recurrence (IBTR). Minimum follow up for living \& event-free patients is 3 yrs.; median follow up time of the whole analyzed patient population is 5.4 yrs. (range: 1.8-11.4 yrs).

Results: Two hundred fifty-one patients were accrued from 2004 to 2013. All patients underwent local excision of the primary tumor to negative margins. Four patients failed in the ipsilateral breast after a median time of 3.2 years (range: 1.7-5.7 yrs) for a 5-year IBTR-free survival of 98.7\% (95\%Cl: 97.3\%-100\%). IBTR-free survival was significantly higher for patients with invasive cancer than for patients with intraductal carcinoma $(p=0.036)$. Within patients with invasive tumors, no clear trends or associations were detected between IBTR and age, grading, molecular subtype, pT or pN stage. At 5 years, the actuarial rates of GR2 fibrosis and GR2+ teleangectasia are 2.4\% (95\%Cl: 0-6.5\%) and 7.1\% (95\%Cl: $0.4-13.7 \%$ ), respectively. Cosmesis was scored as excellent/good by $\approx 95 \%$ of patients and $\approx 60 \%$ of clinicians.

Conclusions: Hypo-WBI in 3 weeks allows excellent oncologic outcomes for invasive breast cancer after conservative surgery. Patients with intraductal carcinoma should be treated with Hypo-WBI only within a controlled study.
\end{abstract}

Trial registration: IRE-IFO Ethical and Scientific Committee (cod. RS61/04).

Keywords: Breast neoplasms, Breast carcinoma in situ, Radiotherapy, Dose hypofractionation, Local neoplasm recurrence

\section{Background}

Breast cancer is the most commonly diagnosed malignancy in females in the US, with almost 250,000 women diagnosed per year [1]. A large proportion of patients presents with early-stage disease and is candidate for breast-conserving therapy. Postoperative whole-breast irradiation (WBI) is the standard of care for these patients [2]. Conventionally fractionated regimens traditionally deliver WBI over approximately 5 weeks in $25-28$ fractions to doses of 45-50 Gy. Further improvements in

\footnotetext{
* Correspondence: giuseppe.sanguineti@ifo.gov.it

${ }^{1}$ Departments of Radiation Oncology, Regina Elena National Cancer Institute, Via Elio Chianesi 53, 00144 Rome, Italy

Full list of author information is available at the end of the article
}

local control rates can be achieved for many patients with additional dose of radiation to a limited volume of the breast ("boost") [3] extending the treatment course by approximately 1 to 2 weeks to an overall treatment time of 6 to 7 weeks. Instead of following WBI completion, the boost can be delivered at each fraction as 'simultaneous integrated boost' [4] thus avoiding further treatment extension beyond WBI.

In attempt to improve efficiency, cost of care delivery as well as patient logistics, alternate techniques and dose scheduling have been investigated. Randomized clinical trials in Canada and the UK have demonstrated that shorter treatment regimens ( 3 to 4 weeks) may be as safe and effective as conventional schedules [5-7]. However, 
there has not been significant adoption of hypofractionated WBI (Hypo-WBI) in the United States [8]. Since the above trials have selected low-risk patients, one concern is whether Hypo-WBI can be offered to patients at higher risk of in-breast failure [4]. Other concerns potentially limiting the use of Hypo-WBI are the use of a boost and its timing relative to WBI [4]. None of the prospective trials for Hypo-WBI systematically delivered a tumor bed boost; in the START A and B trials it was left to the Institutional policy, so it was delivered in only a fraction of patients and in a nonrandomized fashion. Moreover, the use of a sequential boost of 1-2 weeks after WBI extends the overall treatment time reducing the potential timesaving benefit from hypofractionation.

In 2004 we designed a novel treatment schedule of Hypo-WBI in which therapy was completed in 11 fractions over 3 weeks inclusive of a sequential single-fraction boost. After a preliminary phase I part [9], in the present study we report the outcomes of 251 patients at a minimum follow up of 3 years.

\section{Methods \\ Study description}

This is a prospective phase II study evaluating the efficacy of a novel Hypo-WBI schedule. Inclusion criteria were: age $>18$ years; pathologically proven breast carcinoma undergone complete local excision within a breast conserving strategy to negative margins (R0); pathological primary tumor stage up to pT2 (pTis-pT2); pathological nodal stage up to pN1a (pNx-pN1a); no distant metastases (M0); no previous local surgical or radiation treatment; no previous chemotherapy; specific informed consent. The study was approved by the local Institutional Ethical and Scientific Committee (cod. RS61/04). All patients provided a written informed consent.

The study opened to accrual in October 2004 and was initially offered only to selected patients unable to undergo conventional adjuvant radiotherapy in 6-6.5 weeks due to logistic reasons or refusal [9]. This pilot phase lasted until March 2006, when 39 patients had been accrued [9]. After preliminary toxicity data showed its feasibility, this schedule has been offered to all patients operated conservatively for early breast cancer at our Institution and satisfying the above criteria since June 2008.

\section{Immunohistochemistry data}

Histologic grade was scored according to the Nottingham system. Estrogen receptor (ER) status, progesterone receptor (PR) status and proliferation rate (ki67) were assessed throughout immunohistochemistry (IHC). Human epidermal growth factor receptor 2 (HER2) status was initially assessed with IHC. Tumors were considered HER2+ if they received a score of $3+$ on $\mathrm{IHC}$ staining or if they received a score of $2+$ by IHC staining and showed HER2 amplification based on silver-enhanced in situ hybridization (SISH) [10]. Tumors with scores of $2+$ by IHC staining in the absence of SISH amplification were considered HER2 negative.

Patients were categorized based on the receptor status of their primary tumor: luminal A (ER+ or PR+ and HER2and ki67 $<20 \%$ ), luminal B (ER+ or PR+ and HER2+ or ki67 $\geq 20 \%$ ), HER2-enriched (ER- and PR- and HER2+), and triple negative/basal (ER- and PR- and HER2-).

\section{Radiotherapy technique}

The details of radiotherapy technique have been reported previously [9]. Briefly, Hypo-WBI consisted of 34 Gy in 10 daily fractions over 2 weeks to the whole breast, followed by a single fraction of $8 \mathrm{~Gy}$. When the schedule was designed no information was available on the (acute) tolerance to Hypo-WBI. Therefore, one-week gap between the end of Whole Breast irradiation and the boost was planned in order to allow the recovery of normal breast tissues from Hypo-WBI. Despite the preliminary excellent results in terms of acute toxicity [9], the gap was maintained for logistic reasons and it became part of our institutional practice. The regional lymph nodes were not intentionally targeted. Whole breast parenchyma was covered by two tangential fields, $6 \mathrm{MV}$ photon beams 3D-CRT. Wedge compensation was used to ensure a uniform dose distribution to the target volume within $-5 \%$ and $+7 \%$. Moreover, $95 \%$ of the prescribed dose had to be received by at least $95 \%$ (goal) of the planning target volume (PTV), or by $90 \%$ of the PTV as acceptable variation; while maintaining a maximum lung depth $\leq 2.5 \mathrm{~cm}$.

The boost dose of 8 Gy (prescribed to the $90 \%$ reference isodose) was administered via a 6 to $12 \mathrm{MeV}$ appositional electron field.

\section{Study endpoints}

The primary endpoint of the present study is freedom from ipsilateral breast tumor recurrence (IBTR). Local tumor relapse was defined as any breast quadrant local relapse. Any ipsilateral regional relapse outside the radiotherapy target volume was excluded from the analysis of local relapse.

This study was designed to assess the 5-year IBTR rate. On the basis of available literature at the time of the design of the study we assumed a $5 \%$ rate [3]; a sample size of 250 patients would have been able to estimate this percentage with a standard error of $1.38 \%$.

Secondary endpoints include freedom from contralateral breast tumor recurrence (CBTR), from distant metastases (DM) and from second (non-breast) primary tumors. Progression free survival includes any of the above events as well as death due to inter-current causes.

Late toxicity was scored according to the LENT/SOMA [11] and CTCAE v4.0 [12] scales as appropriate. The highest 
level of reaction in any quadrant of the treated breast was considered the final grade. Toxicity grades could be pooled and dichotomized in order to perform further analysis as detailed below. Cosmesis was scored independently by two observers according to the Harvard scale after reviewing the pictures at the last follow up [13].

\section{Statistical analysis}

Distributions of covariates between samples were analyzed by contingency tables (chi square test) and by nonparametric tests (Mann U Whitney). Actuarial curves are computed with the Kaplan-Meier method from the last day of radiotherapy. Patients were still evaluable for localregional relapse after distant relapse, but were censored at date of death. Estimates of 5-year events were calculated (with 95\% Confidence Intervals-CIs). The log-rank test was used to compare survival distributions. Cox proportional hazard regression model was used to estimate hazard ratios (HR) and their 95\% confidence intervals. Within the subgroup of invasive tumors, selected covariates were investigated at univariate analysis for a possible association with the time to IBTR: age (continuum); grading (G3 vs others); primary tumor stage (pT1 vs pT2); nodal stage (pN0 vs pN1a); molecular profile (Luminal vs Her2 enriched vs Triple negative). Significance was claimed for $p$ values below 0.05. Estimation of the 95\% confidence intervals for proportions was done with the Wilson score method. Inter-rater agreement for was estimated through the Cohen's kappa $(\kappa)$. The $\kappa$ value indicates no $(\kappa=0)$, slight $(\kappa=0-0.2)$, fair $(\kappa=0.2-0.4)$, moderate $(\kappa=0.4-0.6)$, substantial $(\kappa=0.6-0.8)$ or almost perfect $(\kappa=0.8-1)$ agreement.

Minimum follow up for living \& event-free patients is 3 yrs.; median follow up time of the whole analyzed patient population is 5.4 yrs. (range: $1.8-11.4 \mathrm{yrs}$ ).

\section{Results}

Patients \& treatment

From October 2004 to August 2013, 251 patients were accrued. Selected patient, tumor and treatment characteristics are reported in Table 1. Mean age is 61.1 yrs. (SD: 12.2). Overall, 52 (20.7\%), 58 (23.1\%), 67 (26.7\%) and $44(17.5 \%)$ patients were younger than 50 years, had pT2 primary tumors, had poorly differentiated tumors (G3), were nodal positive (pN1a), respectively. Slightly more than $10 \%$ of patients had in-situ ductal carcinoma (pTis). Of them, 13 (46.8\%) received also adjuvant hormonal treatment. All patients underwent local excision of the primary tumor to negative margins. Twenty patients did not undergo surgical staging of the axilla (pNx), including 16 patients with DCIS, 1 patient with pT1mic, 2 patients with pT1c and 1 patient with pT2 disease. All patients with pN1a disease underwent dissection of at least levels I and II of the ipsilateral axilla.
Table 1 Selected characteristics of the patients, tumors and treatments

\begin{tabular}{|c|c|c|c|}
\hline Covariate & Stratification & $\mathrm{N}$ & $\%$ \\
\hline \multirow[t]{2}{*}{ Tumor Location } & Superior Half & 189 & 75.3 \\
\hline & Inferior Half & 62 & 24.7 \\
\hline \multirow[t]{5}{*}{ Pathology } & DCIS & 28 & 11.2 \\
\hline & Ductal & 202 & 80.5 \\
\hline & Lobular & 10 & 4.0 \\
\hline & Mixed & 5 & 2.0 \\
\hline & Other & 6 & 2.4 \\
\hline \multirow[t]{4}{*}{ Grading } & G1 & 34 & 13.5 \\
\hline & $\mathrm{G} 2$ & 136 & 54.2 \\
\hline & G3 & 67 & 26.7 \\
\hline & Gx & 14 & 5.6 \\
\hline \multirow[t]{3}{*}{ Estrogen Receptors } & Negative & 39 & 15.5 \\
\hline & Positive & 210 & 83.7 \\
\hline & Unknown & 2 & 0.8 \\
\hline \multirow[t]{3}{*}{ Progesterone Receptors } & Negative & 59 & 23.5 \\
\hline & Positive & 190 & 75.7 \\
\hline & Unknown & 2 & 0.8 \\
\hline \multirow[t]{2}{*}{ Menopausal Status } & Premenopausal & 70 & 27.9 \\
\hline & Postmenopausal & 181 & 72.1 \\
\hline \multirow[t]{3}{*}{ Axilla staging } & SNB(OSNA method $[33,34])$ & 143 & 57.0 \\
\hline & AND & 88 & 35.0 \\
\hline & None & 20 & 8.0 \\
\hline \multirow[t]{6}{*}{ pT Stage } & DCIS & 28 & 11.2 \\
\hline & pT1mic & 4 & 1.6 \\
\hline & pT1a & 15 & 6.0 \\
\hline & pT1b & 37 & 14.7 \\
\hline & pT1c & 109 & 43.4 \\
\hline & pT2 & 58 & 23.1 \\
\hline \multirow[t]{4}{*}{ pN Stage } & $\mathrm{pNO}$ & 180 & 71.7 \\
\hline & pNmic & 7 & 2.8 \\
\hline & pN1a & 44 & 17.5 \\
\hline & $\mathrm{pNx}$ & 20 & 8.0 \\
\hline \multirow[t]{4}{*}{ Adjuvant tmt } & None & 20 & 8.0 \\
\hline & Hormones & 132 & 52.6 \\
\hline & Chemotherapy & 31 & 12.4 \\
\hline & Chemotherapy + Hormones & 68 & 27.1 \\
\hline
\end{tabular}

Abbreviations: SNB sentinal nodal biopsy, OSNA one step nucleic acid amplification, AND axillary nodal dissection, DCIS Ductal carcinoma in situ, $p T$ Primary tumor stage at pathology, $p N$ Nodal stage at pathology, tmt treatment

Molecular profile was assessed in the 223 patients with infiltrative lesions (Table 2). Of the 41 patients with HER2 positive status, $31(75.6 \%)$ received Herceptin as part of their adjuvant systemic treatment. Overall, of 99 patients undergoing adjuvant chemotherapy, most patients (86.8\%) 
Table 2 Molecular profiling of patients with invasive breast cancer $(N=223)$

\begin{tabular}{llll}
\hline Covariate & Stratification & N & $\%$ \\
\hline Ki-67 & $<20 \%$ & 141 & 63.2 \\
& $\geq 20 \%$ & 81 & 36.3 \\
HER2 status & Unknown & 1 & 0.4 \\
Molecular groups & Negative & 182 & 81.6 \\
& Positive & 41 & 18.4 \\
& Luminal A & 127 & 57.0 \\
& Luminal B & 59 & 26.5 \\
& HER2 enriched & 17 & 7.6 \\
& Triple Negative & 19 & 8.5 \\
& Unclassified & 1 & 0.4 \\
\hline
\end{tabular}

Abbreviations: HER2 Human epidermal growth factor receptor 2

received anthracycline- and/or taxane-based chemotherapy. None of the patients underwent neoadjuvant chemotherapy.

All patients completed the planned radiation treatment including the boost. Mean treatment duration (including the boost) was 2.9 weeks (95\% CI: $2.5-3.3$ weeks). The median PTV volume of the breast was $549 \mathrm{~cm}^{3}$ (range $113-2118 \mathrm{~cm}^{3}$ ). WBI treatment plans were normalized to intentionally keep the maximum dose below $107 \%$ of the prescribed dose. Nevertheless the median volume receiving a total dose higher than 107\% (V36.4Gy) of the prescription dose (34 Gy) was less than $0.01 \mathrm{~cm}^{3}$ (range: $0.0-$ $5.3 \mathrm{~cm}^{3}$ ). Therefore, the median prescribed dose was 33.8 Gy (range: 32.5-34.4 Gy). The median mean lung dose was $2.7 \mathrm{~Gy}$ (range: 0.5-6.4Gy). For left-sided tumors, the median mean heart dose was $0.8 \mathrm{~Gy}$ (range: $0.2-3.6 \mathrm{~Gy})$ and the median heart V3 Gy was $7.3 \mathrm{~cm}^{3}$ (range: $0.0-148.5 \mathrm{~cm}^{3}$ ).

\section{Oncologic outcomes}

Four patients developed a local failure after a median time of 38.4 months. Estimated 5-yr. IBTR-free survival is $98.7 \%$ (95\%CI: $97.3-100 \%$ ) (Fig. 1). All observed local

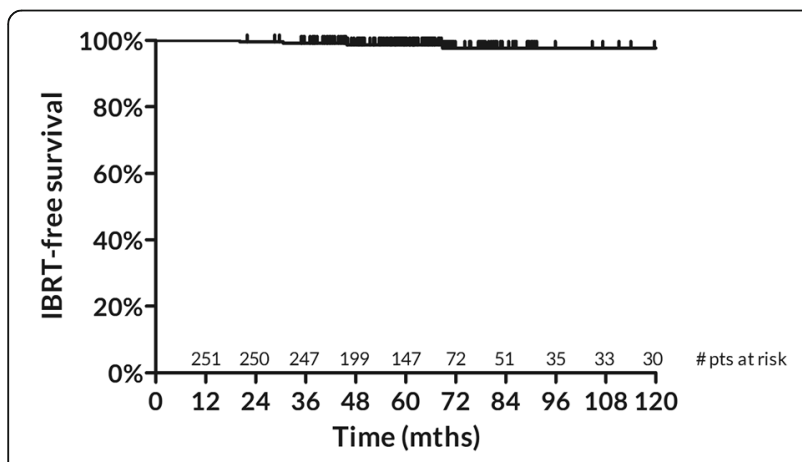

Fig. 1 IBTR-free survival for the overall population failures were infiltrative and in the original quadrant. Selected characteristics of the patients developing a local failure are reported in Table 3.

IBTR-free survival was significantly higher for patients with invasive cancer at diagnosis than for patients with intraductal carcinoma (5-yr. IBTR-free survival 99.1\%, 95\% CI: $97.9-100 \%$ and 95.2\%, 95\%CI: $86.2-100 \%$, respectively, $p=0.036$, Fig. 2).

Within invasive tumors, no clear trends or associations were detected between the time to IBTR and age (continuum) $(p=0.362)$, grading $(p=0.617)$, primary tumor stage at pathology $(\mathrm{pT} 2$ vs $\mathrm{pT} 1)(p=0.471)$ or lymph node stage at pathology ( $\mathrm{pN} 0$ vs $\mathrm{pN} 1 \mathrm{a})(p=0.89)$ and molecular profile (Triple negative vs HER-2 enriched vs Luminal A-B) $(p=0.70)$.

Four patients developed distant metastases and, of them, 2 died of disease. Five-year DM-free survival is 98.1\% (95\%CI: 97.9-98.3\%). Five patients developed a contralateral breast cancer, 4 infiltrative and 1 ductal carcinoma in situ after a median time of 45.6 months (range: 9.6-80.4 months). Five-year CBRT-free survival is $98.7 \%$ (95\%CI: $97.3-100 \%)$. Thirteen patients developed a second primary tumor (which was lethal in 3) and 3 additional patients died of intercurrent disease. Five-year overall and progression free survivals are 96.7\% (95\%CI: $94.2-99.2 \%$ ) and $91.6 \%$ (95\%CI: $87.9-$ 95.3\%), respectively.

\section{Toxicity \& Cosmesis}

Five patients developed peak grade 2 fibrosis (CTCAE v4.0) of the treated breast. Eighteen patients developed skin teleangectasia (LENT/SOMA): grade (GR) 2 and GR3 in 11 and 7 patients respectively. Figure 3 shows the time to GR2 fibrosis and GR2+ teleangectasia, respectively. At 5 years, the actuarial rates of GR2 fibrosis and GR2+ teleangectasia are $2.4 \%$ (95\% CI: $0-6.5 \%)$ and $7.1 \%(95 \% \mathrm{CI}$ : 0.4-13.7\%), respectively. No other GR2+ reaction has been recorded.

Cosmesis was assessed at a mean (SD) time of 63.7 (26.5) months after treatment end on $223(88.8 \%)$ of patients. Results are illustrated in Fig. 4. Regarding physician-reported scores, slightly more than $60 \%$ of patients were judged to have an excellent or good cosmetic appearance by either observer. Conversely, $\approx 15 \%$ of patients were considered to have a poor cosmetic outcome. The inter-observer agreement is substantial $(\kappa=0.648, p<0.001)$. According to patientreported scores, the majority of patients (94.2\%) felt to have an excellent or good cosmetic outcome. There was no correlation between patient and physician scores $(\kappa=-0.007$ and $\kappa=0.022$ between patients and observer 1 and between patients and observer 2, respectively). 
Table 3 Selected patient, tumor and treatment characteristics for patients who developed a IBTR

\begin{tabular}{|c|c|c|c|c|c|c|c|c|c|c|c|}
\hline Initials & $\begin{array}{l}\text { Age } \\
\text { (years) }\end{array}$ & Grade & Path & pT & $\mathrm{pN}$ & $\begin{array}{l}\text { ER/PR } \\
\text { status }\end{array}$ & Ki-67 & $\begin{array}{l}\text { HER2 } \\
\text { status }\end{array}$ & $\begin{array}{l}\text { Molecular } \\
\text { Group }\end{array}$ & Systemic tmt & $\begin{array}{l}\text { Time to IBTR } \\
\text { (months) }\end{array}$ \\
\hline F.R. & 45 & 3 & $D$ & is & $x$ & NA & NA & NA & NA & None & 68.8 \\
\hline A.A. & 68 & 3 & D & is & 0 & Positive & NA & NA & NA & None & 45.9 \\
\hline R.B. & 50 & 2 & D & 2 & $1 a$ & Positive & $5 \%$ & Negative & Luminal A & Chemo-horm & 30.6 \\
\hline D.M. & 56 & 2 & D & 2 & 0 & Positive & $40 \%$ & Positive & Luminal B & Chemo-horm & 20.2 \\
\hline
\end{tabular}

Abbreviations: IBTR ipsilateral breast tumor recurrence, HER2 Human epidermal growth factor receptor $2, p T$ Primary tumor stage at pathology, $p N$ Nodal stage at pathology, tmt treatment, $D$ invasive ductal carcinoma

\section{Discussion}

The results of the present prospective single-Institution study confirm Hypo-WBI to be an effective option after breast conserving surgery for early stage invasive breast carcinoma. Our 5-year estimate of IBTR is comparable to the ones obtained within selected phase III studies at a similar length of follow up (Fig. 5). Local recurrence is usually higher between years 3 and 5 of follow up, with $2 / 3$ of local failures taking place within 5 yrs. [14]. Therefore, even if further IBTR events are to be expected, we believe that our results are reasonably mature with regard to this endpoint.

The Cox proportional hazard regression of both START-P and START-A trials allowed a direct estimate of the radiosensitivity $(\alpha / \beta$ ratio) for tumor local recurrence at 3.5 Gy (95\%CI: 1.3-5.7 Gy) [15]. When applied to the present schedule, 34 Gy in 10 fractions would be equivalent to 42.6 Gy (95\%CI: 40.2-48.4 Gy) delivered at 2.0 Gy fractions (EQD2 Gy), assuming no impact of treatment time. In other words, disregarding the effect of shortening the treatment time from 5 to 2 weeks, 34. Gy in 10 fractions would correspond to $42.6 \mathrm{~Gy}$ in 21.3 fractions. When the treatment time is factored in by considering a daily dose of 0.60 Gy necessary to compensate for each saved day of treatment $($ Dprolif $=$ $0.60 \mathrm{~Gy} /$ day, $95 \% \mathrm{CI}: 0.10$ to $1.18 \mathrm{~Gy} /$ day) [16], the EQD2 Gy for WBI jumps to 55.2 Gy (95\%CI: 50.5$65 \mathrm{~Gy}$ ). Interestingly, despite similar (but not identical)

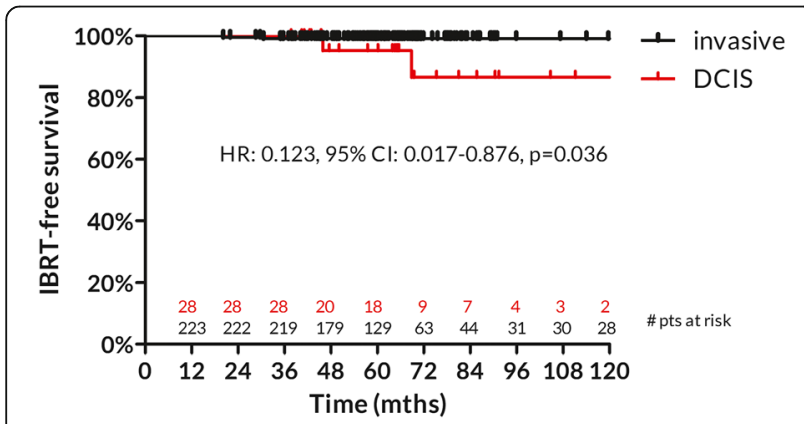

Fig. 2 IBTR-free survival for invasive and ductal carcinoma in situ (DCIS). Considering the DCIS subgroup as the reference one, the Hazard Ratio (HR) of IBTR for the invasive group is shown entry criteria, Fig. 5 shows that the most favorable IBTR rates have been achieved within studies investigating schedules that shorten WBI by $2-3$ weeks such as the IRE, OCOG and START B ones.

The role of a boost dose to the tumor bed in the setting of hypofractionated WBI remains unclear. In 2011, the ASTRO task force recommended not to use hypofractionated RT when a tumor bed boost was thought to be indicated due to the lack of data at that time [17]. In the earlier part of START-P trial, there was a subrandomization between boost (14 Gy in 7 fractions, 364 pts) and no boost (359 pts) after WBI. Unfortunately, no clinical results have been disclosed [14]. In the following START trials the use of a boost (10 Gy/5 fractions) after WBI was left to each Institution policy and, overall, $\approx 50 \%$ of patients received it. Conversely, in the OCOG trial, excellent IBTR rates have been achieved without a boost [7].

In our experience a tumor bed boost was always delivered regardless clinical/tumor factors and thus it became integral part of the treatment schedule. By applying the same parameters as above, 8 Gy in single fraction corresponds to an EQD2 Gy of 9.2 Gy (95\% CI: 7.4-11.0 Gy) for a cumulative tumor bed dose in 3 weeks of $61.1 \mathrm{~Gy}$ (95\%CI: 44.8-77.4 Gy). In other words, it is estimated that, on average, the tumor bed boost adds only 5 more

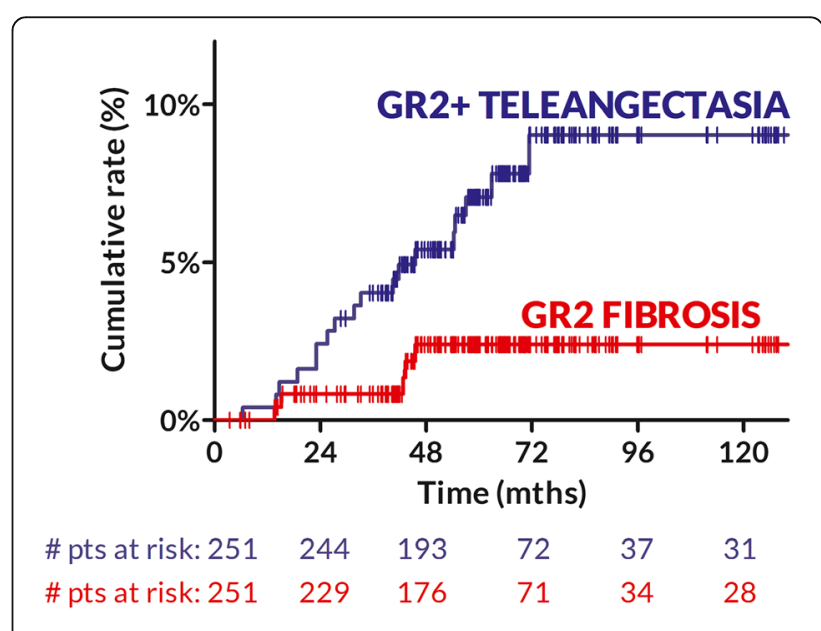

Fig. 3 Cumulative rates of both GR2 fibrosis and GR2+ teleangectasia 


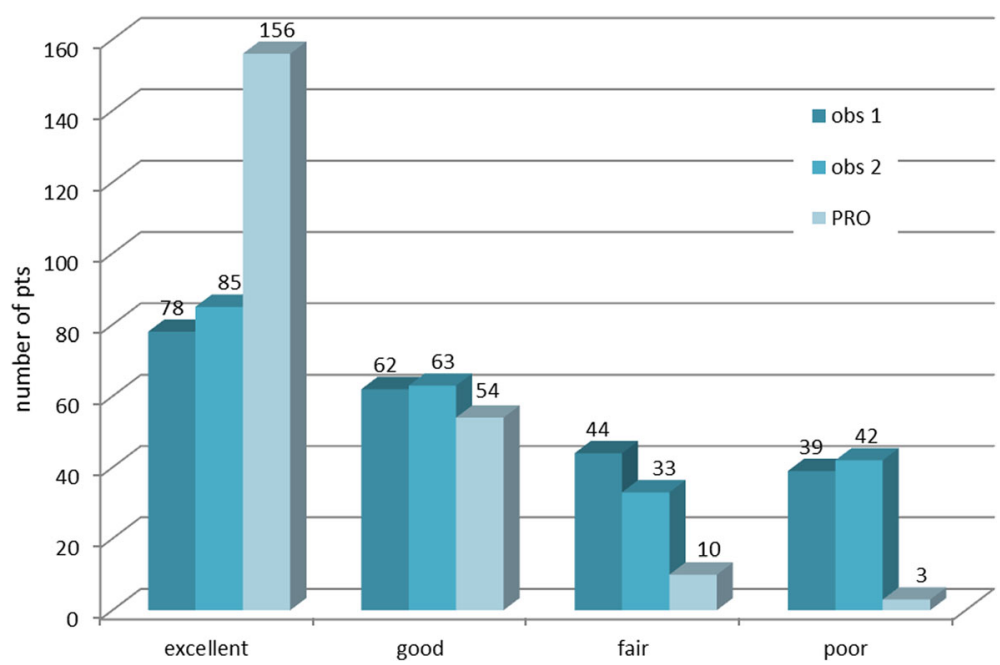

Fig. 4 Cosmetic outcomes according to both clinicians (obs 1 and obs2: observer 1 \& 2) and patients (PRO: patient reported outcomes)

Gy at 2 Gy due to the week break after WBI. Since the slope of the dose-response at the IBTR rates observed here is very shallow [16], we doubt that such a limited increase in the total dose had a large effect on local control. Therefore, one option would be to discontinue the systematic use of the boost, reserving it only for selected patients (i.e. those with high grade tumors and those with an intraductal component). Moreover, in this case, in order to maintain a reasonably high biological

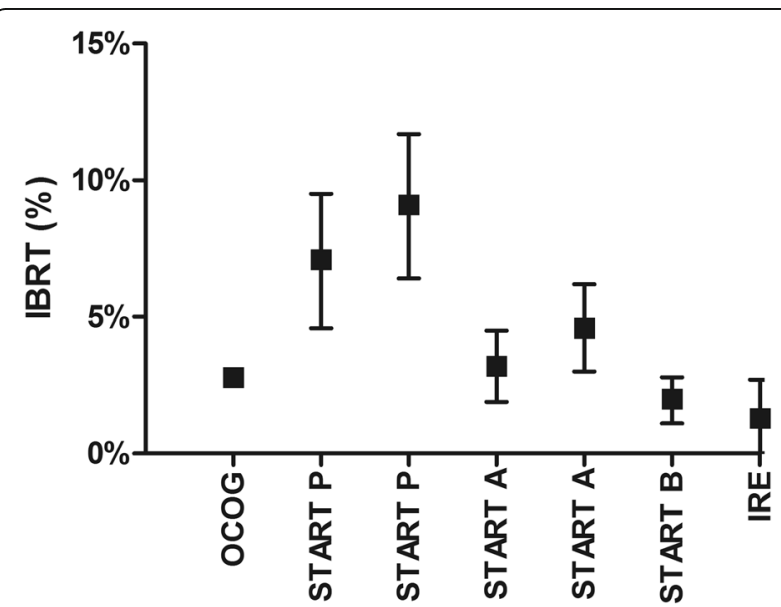

Fig. 5 Five-yr. IBTR $(95 \% \mathrm{Cl})$ from randomized controlled studies along with the present study (Invasive ca only)(IRE: Regina Elena Institute). The experimental arms of each study were as follow: OCOG (Ontario Clinical Oncology Group Trial), 42 Gy/16 fractions/3.2 wks, median follow up 5.75 yrs., 622 pts. [5]; START P

(Standardization of Radiotherapy Pilot) trial, 39 Gy/13 fractions/5 wks and $42.9 \mathrm{~Gy} / 13$ fractions/5 wks, median follow up 9.7 yrs., 474 and 466 patients, respectively [12]; START A trial, 39 Gy/13 fractions/5 wks and $41.6 \mathrm{~Gy} / 13$ fractions/5 wks, median follow up $5.1 \mathrm{yrs.}, 737$ and 750 patients, respectively [4]; START B trial, 40 Gy/15 fractions/3 wks, median follow up 6.0 yrs., 1110 patients [3] effective dose, it might be worth to consider delivering the boost right after WBI (avoiding any break) or to investigate a concurrent boost delivery technique ('simultaneous integrated boost') as others have successfully explored [4].

Multiple studies have suggested that the molecular tumor profile predicts for IBTR after conventional RT $[18,19]$. However, there are limited data on the efficacy of hypofractionation by breast cancer molecular subtype [20]. Post-hoc analysis on 989 patients $(80 \%)$ accrued within the OCOG study for whom formalin-fixed paraffin-embedded tumor block were available, showed that molecular subtype was the only predictor of local recurrence at multivariable analysis. The 10 -yr. IBTR rates were $4.5 \%$ for luminal A and basal-like, 7.9\% for luminal B and $16.9 \%$ for HER2 enriched tumors $(p<0.01)$ [20]. Moreover, no evidence of an interaction between RT fractionation (hypofractionated or standard) and molecular subtype was observed for IBTR [18]. Contrary to the OCOG and other studies $[18,19]$, in the present one trastuzumab was considered for patients with HER2 tumors, and this may have compensated for the poorer prognosis of HER2 enriched tumors [21]. A longer follow up on a larger number of patients is needed to confirm this finding.

A recent meta-analysis tried to clarify the role of hypofractionation for ductal carcinoma in situ (DCIS) [22]. Since DCIS tumors were not included in any prospective randomized study on hypofractionation, the Authors analyzed 4 observational studies comparing IBTR rates between patients who received standard vs hypofractionated RT (2534 pts) finding no difference in local recurrence rate by fractionation despite a trend in favor of hypofractionation (OR: 0.78, 95\% CI: 0.58-1.03, $p=0.08$ ) [22]. An ongoing phase III study (NCT00470236) by the 
Trans-Tasman Radiation Oncology Group is trying to clarify the issue. In the meantime, the present data raise caution when treating patients affected by DCIS with hypofractionation.

Teleangectasia and fibrosis are the most prevalent late side effects after breast irradiation [23]. Fibrosis incidence is maximal during the first few years after treatment and then plateaus [24] while skin teleangectasia shows a progressive onset at least up to 10 yrs. after treatment [25]. Therefore, the rate of mild fibrosis observed in the present study $(2.4 \%$ with an upper $95 \%$ limit of $6.5 \%)$ along with the lack of severe (GR3) toxicity seems particularly favorable and similar if not better than historical controls at comparable time-points [26]. Since the risk of fibrosis seems related to the maximal dose delivered during whole breast irradiation rather than volume parameters [24], we believe that the fact that our WBI treatment plans were highly homogeneous may have had a major role in reducing its occurrence.

It has been shown that, for a given fractionation schedule, fibrosis and telangiectasia develop independently within the same patient [27] and have different predictors [28, 29]. Accordingly, the estimated 5-yr. rate of GR2+ teleangectasia (7.1\%, upper 95\% limit: 13.7\%) was higher than GR2 fibrosis (Fig. 3), though it seems comparable to the rate (12.4\%) reported in the boost arm of the Lyon trial at the same time point [28]. We have previously shown that selected boost parameters (area and electron energy) are independently correlated to the risk of GR2+ telangiectasia [9]. Therefore, it is possible that changing boost strategy (both indication and technique, as previously discussed) will reduce the prevalence of GR2+ teleangectasia.

Similar to other studies [30,31], the overall concordance between clinicians and patients in scoring toxicity was low. Moreover, we found that, on average, patient perception of toxicity is more optimistic than physician one. Overall, the vast majority of patients felt to have had an excellent or good cosmetic outcome, while the rate dropped to $\approx 60 \%$ when scored by the physician. Though using different scales, others have reported similar rates for physician reported outcomes. In the OCOG study, cosmetic outcome according to the EORTC Cosmetic rating System on 448 patients treated within the short arm was excellent or good in $77.9 \%$ of patients at 5 years [7]. In the START P trial at a minimum follow up of 5 years, physician-scored excellent/ good cosmetic results were reported in $54.6 \%$ and $37.9 \%$ of patients after 39 Gy and 42.6 Gy, respectively [32].

\section{Conclusion}

In conclusion, the results of this single-Institution prospective study on 251 patients at a minimum follow up of 3 yrs. show that Hypo-WBI is associated with a very low risk of both IBTR and long term toxicity as well as with good to excellent cosmetic outcomes in most of the patients. Though both oncologic and morbidity outcomes will be continuously monitored, 34 Gy in 10 fractions to the whole breast followed by a single dose boost are currently offered as standard treatment at our Institution for all the patients with invasive cancer after breast conservation surgery who fit the above inclusion criteria. The present results also suggest that the delayed singlefraction boost may be safely omitted, and this in turn may reduce the risk of skin teleangectasia, though this deserves prospective confirmation among properly selected (low risk) patients. Finally, for patients with DCIS, the present data do not support treatment with Hypo-WBI outside a controlled/research study.

\begin{abstract}
Abbreviations
3D-CRT: Three-dimensional-conformal Radiotherapy; AND: Axillary nodal dissection; CBTR: Contralateral breast tumor recurrence; D: Invasive ductal carcinoma; DCIS: Ductal carcinoma in situ; DM: Distant metastases; EQD2 Gy: Equivalent dose delivered at 2.0 Gy fractions; ER: Estrogen receptor; HER2: Human epidermal growth factor receptor 2; Hypo-

WBI: Hypofractionated WBI; IBTR: Ipsilateral breast tumor recurrence; IHC: Immunohistochemistry; ki67: Proliferation rate; OSNA: One step nucleic acid amplification; pN: Nodal stage at pathology; PR: Progesterone receptor; pT: Primary tumor stage at pathology; SD: Standard deviation; SISH: Silver-enhanced in situ hybridization; SNB: Sentinal nodal biopsy; tmt: Treatment; WBI: Whole-breast irradiation
\end{abstract}

\section{Acknowledgements}

This work has been partially found by the NATO (project n. G4815/2014 Programme SPS), which is greatly acknowledged.

\section{Authors' contributions}

PP: Conceptualization, methodology, guarantor of the data quality, writing (reviewing/editing), formal analysis, visualization, approval of the manuscript. CG: Data curation, approval of the manuscript. AF: Data curation, approval of the manuscript. AF: Data curation, approval of the manuscript. Gl: Data curation, approval of the manuscript. VL: Conceptualization, visualization, approval of the manuscript. DG: Methodology, formal analysis, visualization, approval of the manuscript. PV Writing (reviewing/editing), approval of the manuscript. LS: Formal analysis, writing (reviewing/editing), approval of the manuscript. GS: Conceptualization, methodology, writing (first draft), writing (reviewing/editing), formal analysis, visualization, supervision/administration, approval of the manuscript.

\section{Ethics approval and consent to participate}

The study has been approved by the Institutional Ethical and Scientific Committee. All enrolled patients signed an informed consent for study participation.

\section{Consent for publication \\ Not applicable.}

\section{Competing interests}

The authors declare that they have no competing interests.

\section{Publisher's Note}

Springer Nature remains neutral with regard to jurisdictional claims in published maps and institutional affiliations.

\section{Author details}

'Departments of Radiation Oncology, Regina Elena National Cancer Institute, Via Elio Chianesi 53, 00144 Rome, Italy. ${ }^{2}$ Laboratory of Medical Physics and Expert Systems, Regina Elena National Cancer Institute, Rome, Italy.

${ }^{3}$ Departments of Statistics, Regina Elena National Cancer Institute, Rome, Italy. ${ }^{4}$ Departments of Clinical Oncology, Regina Elena National Cancer Institute, Rome, Italy. 
Received: 17 September 2017 Accepted: 16 November 2017 Published online: 27 December 2017

\section{References}

1. DeSantis C, Ma J, Bryan L, Jemal A. Breast cancer statistics, 2013. CA Cance J Clin. 2014;64(1):52-62. https://doi.org/10.3322/caac.21203. Epub 2013 Oct 1. Review

2. Fisher B, Redmond C, Poisson R, Margolese R, Wolmark N, Wickerham L, et al. Eight-year results of a randomized clinical trial comparing total mastectomy and lumpectomy with or without irradiation in the treatment of breast cancer. N Engl J Med. 1989:320:822-8.

3. Bartelink $\mathrm{H}$. The changing landscape in radiotherapy for breast cancer: lessons from long term follow-up in some European breast cancer trials. Radiother Oncol. 2016;121:348-56.

4. Freedman GM, White JR, Arthur DW, Allen Li X, Vicini FA. Accelerated fractionation with a concurrent boost for early stage breast cancer. Radiother Oncol. 2013;106:15-20.

5. START Trialists' Group, Bentzen SM, Agrawal RK, Aird EG, Barrett JM, BarrettLee PJ, et al. The UK standardisation of breast radiotherapy (START) trial B of radiotherapy hypofractionation for treatment of early breast cancer: a randomised trial. Lancet. 2008;371:1098-107.

6. START Trialists' Group, Bentzen SM, Agrawal RK, Aird EG, Barrett JM, BarrettLee PJ, et al. The UK standardisation of breast radiotherapy (START) trial a of radiotherapy hypofractionation for treatment of early breast cancer: a randomised trial. Lancet Oncol. 2008;9:331-41.

7. Whelan T, MacKenzie R, Julian J, Levine M, Shelley W, Grimard L, et al. Randomized trial of breast irradiation schedules after lumpectomy for women with lymph node-negative breast cancer. J Natl Cancer Inst. 2002;94:1143-50.

8. Jagsi R, Falchook AD, Hendrix LH, Curry H, Chen RC. Adoption of hypofractionated radiation therapy for breast cancer after publication of randomized trials. Int J Radiat Oncol Biol Phys. 2014:90:1001-9.

9. Pinnarò P, Soriani A, Landoni V, Giordano C, Papale M, Marsella A, et al. Accelerated hypofractionated radiotherapy as adjuvant regimen after conserving surgery for early breast cancer: interim report of toxicity after a minimum follow up of 3 years. J Exp Clin Cancer Res. 2010;29:9.

10. Piccart-Gebhart MJ, Procter M, Leyland-Jones B, Goldhirsch A, Untch M, Smith I, et al. Trastuzumab after adjuvant chemotherapy in HER2-positive breast cancer. N Engl J Med. 2005;353:1659-72.

11. LENT SOMA scales for all anatomic sites. Int J Radiat Oncol Biol Phys. 1995 31(5):1049-91. No abstract available.

12. https://evs.nci.nih.gov/ftp1/CTCAE/CTCAE_4.03_2010-06-14_QuickReference_ 5x7.pdf. Accessed 21 Nov 2017.

13. Harris JR, Levene MB, Svensson G, Hellman S. Analysis of cosmetic results following primary radiation therapy for stages I and II carcinoma of the breast. Int J Radiat Oncol Biol Phys. 1979;5:257-61.

14. Owen JR, Ashton A, Bliss JM, Homewood J, Harper C, Hanson J, et al. Effect of radiotherapy fraction size on tumour control in patients with early-stage breast cancer after local tumour excision: long-term results of a randomised trial. Lancet Oncol. 2006;7:467-71.

15. Haviland JS, Owen JR, Dewar JA, Agrawal RK, Barrett J, Barrett-Lee PJ, et al. The UK standardisation of breast radiotherapy (START) trials of radiotherapy hypofractionation for treatment of early breast cancer: 10-year follow-up results of two randomised controlled trials. Lancet Oncol. 2013;14:1086-94.

16. Haviland JS, Bentzen SM, Bliss JM. Yarnold JR; START trial management group, Prolongation of overall treatment time as a cause of treatment failure in early breast cancer: An analysis of the UK START (Standardisation of Breast Radiotherapy) trials of radiotherapy fractionation. Radiother Oncol. 2016;12:420-3.

17. Smith BD, Bentzen SM, Correa CR, Hahn CA, Hardenbergh PH, Ibbott GS, et al. Fractionation for whole breast irradiation: an American Society for Radiation Oncology (ASTRO) evidence-based guideline. Int J Radiat Oncol Biol Phys. 2011;81:59-68.

18. Hattangadi-Gluth JA, Wo JY, Nguyen PL, Abi Raad RF, Sreedhara M, Niemierko A, et al. Basal subtype of invasive breast cancer is associated with a higher risk of true recurrence after conventional breast-conserving therapy. Int J Radiat Oncol Biol Phys. 2012;82:1185-91.

19. Voduc KD, Cheang MC, Tyldesley S, Gelmon K, Nielsen TO, Kennecke H. Breast cancer subtypes and the risk of local and regional relapse. J Clin Oncol. 2010;28:1684-91.

20. Bane AL, Whelan TJ, Pond GR, Parpia S, Gohla G, Fyles AW, et al. Tumor factors predictive of response to hypofractionated radiotherapy in a randomized trial following breast conserving therapy. Ann Oncol. 2014;25:992-8.
21. Fabi A, Malaguti P, Vari S, Cognetti F. First-line therapy in HER2 positive metastatic breast cancer: is the mosaic fully completed or are we missing additional pieces? J Exp Clin Cancer Res. 2016;35:104.

22. Nilsson C, Valachis A. The role of boost and hypofractionation as adjuvant radiotherapy in patients with DCIS: a meta-analysis of observational studies. Radiother Oncol. 2015;114:50-5.

23. Archambeau JO, Pezner R, Wasserman T. Pathophysiology of irradiated skin and breast. Int J Radiat Oncol Biol Phys. 1995;31:1171-85.

24. Collette S, Collette L, Budiharto T, Horiot JC, Poortmans PM, Struikmans H, et al. Predictors of the risk of fibrosis at 10 years after breast conserving therapy for early breast cancer: a study based on the EORTC trial 2288110882 'boost versus no boost'. Eur J Cancer. 2008;44:2587-99.

25. Turesson I. Individual variation and dose dependency in the progression rate of skin telangiectasia. Int J Radiat Oncol Biol Phys. 1990;19:1569-74.

26. Hamilton DG, Bale R, Jones C, Fitzgerald E, Khor R, Knight K, et al. Impact of tumour bed boost integration on acute and late toxicity in patients with breast cancer: a systematic review. Breast. 2016;27:126-35.

27. Bentzen $\mathrm{SM}$, Overgaard M. Relationship between early and late normal-tissue injury after postmastectomy radiotherapy. Radiother Oncol. 1991;20:159-65.

28. Romestaing P, Lehingue Y, Carrie C, Coquard R, Montbarbon X, Ardiet JM, et al. Role of a 10-Gy boost in the conservative treatment of early breast cancer: results of a randomized clinical trial in Lyon, France. J Clin Oncol. 1997;15:963-8.

29. Pinnarò P, Giordano C, Farneti A, Strigari L, Landoni V, Marucci L, et al. Impact of sequencing radiation therapy and chemotherapy on long-term local toxicity for early breast cancer: results of a randomized study at 15year follow-up. Int J Radiat Oncol Biol Phys. 2016;95:1201-9.

30. Mukesh MB, Qian W, Wilkinson JS, Dorling L, Barnett GC, Moody AM, et al. Patient reported outcome measures (PROMs) following forward planned field-in field IMRT: results from the Cambridge breast IMRT trial. Radiother Oncol. 2014;111:270-5.

31. Sneeuw KC, Aaronson NK, Yarnold JR, Broderick M, Regan J, Ross G, et al. Cosmetic and functional outcomes of breast conserving treatment for early stage breast cancer. 1. Comparison of patients' ratings, observers' ratings, and objective assessments. Radiother Oncol. 1992;25:153-9.

32. Yarnold J, Ashton A, Bliss J, Homewood J, Harper C, Hanson J, et al. Fractionation sensitivity and dose response of late adverse effects in the breast after radiotherapy for early breast cancer: long-term results of a randomised trial. Radiother Oncol. 2005;75:9-17.

33. Di Filippo F, Di Filippo S, Ferrari AM, Antonetti R, Battaglia A, Becherini F, et al. Elaboration of a nomogram to predict nonsentinel node status in breast cancer patients with positive sentinel node, intraoperatively assessed with one step nucleic amplification: Retrospective and validation phase. J Exp Clin Cancer Res. 2016:35:193.

34. Di Filippo F, Giannarelli D, Bouteille C, Bernet L, Cano R, Cunnick G, et al. Elaboration of a nomogram to predict non sentinel node status in breast cancer patients with positive sentinel node, intra-operatively assessed with one step nucleic acid amplification method. J Exp Clin Cancer Res. 2015;34:136.

\section{Submit your next manuscript to BioMed Central and we will help you at every step:}

- We accept pre-submission inquiries

- Our selector tool helps you to find the most relevant journal

- We provide round the clock customer support

- Convenient online submission

- Thorough peer review

- Inclusion in PubMed and all major indexing services

- Maximum visibility for your research

Submit your manuscript at www.biomedcentral.com/submit
) Biomed Central 